\title{
AN ONTOLOGY APPROACH FOR THE INTEGRATION OF VARIOUS VEHICLE SERVICES IN V2X COMMUNICATION
}

\author{
Minwoo $\mathrm{Ryu}^{1}$ and Si-Ho Cha ${ }^{2 *}$ \\ ${ }^{1}$ Service Laboratory Institute of Convergence Technology, KT R\&D Center, \\ Seoul, South Korea \\ ${ }^{2}$ Department of Multimedia Science, Chungwoon University, Incheon, \\ South Korea \\ ${ }^{1}$ mw.ryu@kt.com, ${ }^{2}$ shcha@ chungwoon.ac.kr
}

\begin{abstract}
Through numerous vehicle technologies advances, vehicular ad hoc network (VANET) applications have emerged as a new paradigm for the automotive industries. VANET is a special kind of mobile ad hoc network (MANET) which is a revolutionary technology that allows vehicles to be interconnected with each other using VANET communications protocols such as vehicle to vehicle $(\mathrm{V} 2 \mathrm{~V})$, vehicle to infrastructure (V2I), vehicle to pedestrian (V2P), and vehicle to network (V2N). From this communication infrastructure, VANET can provide various services to the connected vehicle and their users. However, each communication protocol cannot interoperate with one another due to their characteristics. Although much research has been proposed to resolve the issues, some important issues have not yet been addressed. To resolve service silo, we provide an ontology for integrating existing vehicle services. To this end, we define the characteristics of each protocol and represent each resource in the ontology. Finally, to show feasibility, we implement the ontology using the protégé tool. Through the test results of this implementation, we can be sure that the vehicle ontology can eventually contribute to integrating various vehicle services in vehicle to everything (V2X) communication.
\end{abstract}

Keywords - V2X, ontology, OWL-S, VANET, service platform

\section{INTRODUCTION}

Vehicular ad hoc network (VANET) is a special kind of mobile ad hoc network (MANET) which has been a growing technological trend in the automobile industry [1] VANET has emerged as a new paradigm and represents a technological revolution so that vehicles could be interconnected with distributed sensor network [2], dedicated short-range communications [3], and next-generation radio communications (LTE [4] and 5G [5]). Accordingly, vehicles in VANET will be able to provide various intelligence services to connecting vehicles and their users according to service scenario of communication methods, such as vehicle to everything (V2X) including vehicle to vehicle $(\mathrm{V} 2 \mathrm{~V})$, vehicle to infrastructure (V2I), vehicle to pedestrian (V2P), and vehicle to network (V2N) [6]. First of all, V2V is used to share information between vehicles where each vehicle could send a short message to other vehicles using the well-known $802.11 \mathrm{p}$ protocol [7]. In particular, it focusses on delivering safety messages. Second, V2I is used to exchange information

Received: May 10, 2019

Reviewed: July 18, 2019

Accepted: August 5, 2019

* Corresponding Author

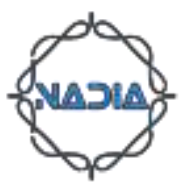


between vehicle and infrastructure so the vehicle can send their information to other systems using the onboard unit (OBU) and roadside unit (RSU). Accordingly, vehicles can provide various services including non-vehicle services (e.g., payment and booking). Third, V2P is used to share information between vehicles and pedestrian to enhance pedestrian safety. In this scenario, safety messages such as traffic sign and vehicle information are sent to the mobile device of pedestrians from OBU or RSU using next-generation radio communications. Finally, V2N is used to utilize the various applications inter-vehicle. To this end, vehicles use public networks using wireless communications as Wi-Fi hotspots [8]. Figure 1 shows the communication methods of V2X communication in VANET.

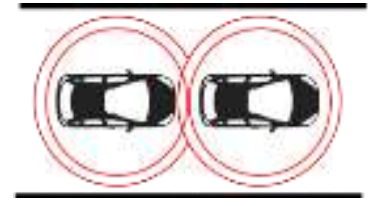

(a)

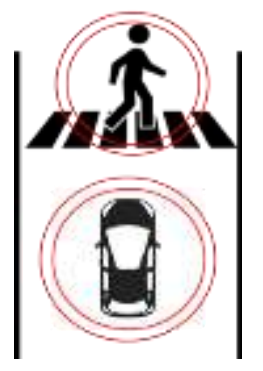

(c)

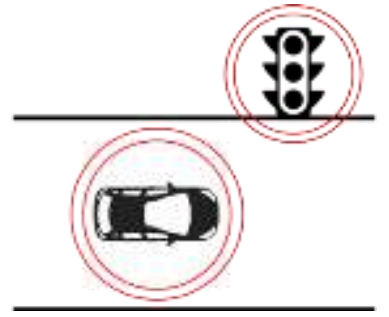

(b)

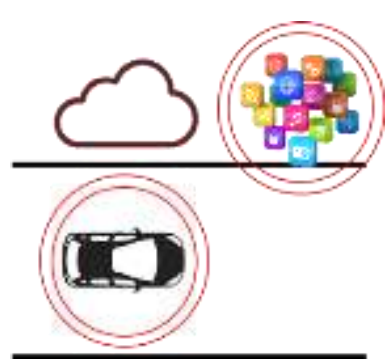

(d)

Fig. 1 V2X communication methods: (from left) vehicle to vehicle (V2V), vehicle to infrastructure (V2I), vehicle to pedestrian (V2P) and vehicle to network (V2N).

Through these advantages of VANET, it could be a key to realizing the potential for providing intelligent vehicle services in an automobile. Due to the characteristics of V2X communications in VANET as shown in Figure 1, it has been difficult to integrate existing vehicle services or newly created services. Although much research has been proposed to resolve these issues, some important issues have not yet been rectified. First, most VANET services provide a push-based service. Due to different characteristics of V2X communications, however, the information should comply with the specific protocols in order to send information to connecting vehicles or others. Second, VANET services have their role according to different scenarios in V2X. In particular, most scenarios focus on the safety of drivers and pedestrian. Accordingly, the applications should be operated on a specific situation before human controls. Finally, most VANET applications operate on their own service platforms or devices which have vertical service architectures. In other words, existing applications cannot interwork with each other.

To resolve these issues, we propose an ontology approach to integrate VANET services on a horizontal service architecture. To this end, we have designed vehicle service ontology based on OWL-S [9] which is an ontological model to describe semantic services. We define resources to use services and binding methods corresponding to each communication in V2X. Finally, to show the feasibility of the proposed ontology, we implement the ontology using protégé. 
The rest of this paper is organized as follows. The existing related work in this research work is introduced in Section 2. The proposed ontology is presented in Section 3. The implementation is introduced in Section 4. Finally, we conclude our remarks in Section 5.

\section{RELATED WORK}

In this section, we introduce existing related work in this research field. VANET is a communication technology for automobiles [10]. Vehicles in VANET are wirelessly connected with each other using multi-hop communication without access to some fixed infrastructure; it is a special kind of mobile ad hoc network (MANET) [11]. However, unlike existing MANET, VANET has high node mobility and rapidly changing network topology. Due to this characteristic, various challenge issues (e.g., link breakage) in VANET have been studied in the last decade [12-14]. Through these efforts, VANET can be a key technology enabling a fully autonomous vehicle. Furthermore, various services in VANET have appeared as new opportunities for the autonomous vehicle.

Silva et. al., proposed an information-driven software-defined vehicular network architecture [15]. In the proposed architecture, they provide content delivery services to vehicular applications. Furthermore, they integrate the concept of existing informationcentric networking (ICN) with the software-designed network (SDN) to the VANET's characteristics.

Grewe et. al., proposed an enhancement of ICN with a semantic descriptor in order to apply it to the vehicular IoT scenario [16]. In this article, they provide a demonstration of the semantic discovery to integrate ICN and design an architecture for the scenario of vehicular networks and vehicular IoT. Finally, they present example domain ontology and a proof of concept implementation using the Name resolution (NRS). From this concept, they introduce a semantic object discovery system in ICN in order to support open context systems.

However, Silva and Grewe use ICN instead of current network architecture. Accordingly, to apply the proposed architectures into real-world situations, we have to change all of the current infrastructures.

Ruta et. al., proposed a novel logic-based framework enabling contextual data management and mining in VANET [17]. In particular, they introduce a distributed collaborative framework for context awareness in vehicular networks based on two main contributions. Furthermore, in the proposed framework, they try information fusion with IoT in order to build a knowledge base. Finally, they use semantic technology to integrate knowledge according to dynamic scenarios. However, they only consider V2V and V2I scenarios in the proposed framework. Hence, we have to furtuer consider various service scenarios in order to apply the framework to V2X.

Park et. al., proposed the recognition prioritization of road environment for supporting autonomous vehicles [18]. They conducted research to determine the priority with which the road infrastructure attributes should be acquired using the AHP (Analytical Hierarchy Process) method.

Bibi et. al., proposed the message dissemination method using an ontology, named the Messaging Ontology for VANET (MOVA) [19]. The proposed ontology aims to enhance the performance of VANET in order to disseminate the message between vehicles. In particular, it focuses on traffic safety-related services. In this article, vehicle, message, road, and occupant are represented as top-level ontology. Furthermore, they use FaCT++ as a reasoner in order to infer the message and situation. However, the proposed ontology does not have enough information to apply the ontology into various service scenarios in V2X.

Recently, many researchers have been trying to provide architectures and methods for integrating various services in V2X. Due to the characteristics of different service scenarios in V2X, however, to integrate various vehicle services, we have to resolve the issues as 
mentioned in Section 1. In this perspective, we provide an ontological approach for the integration of vehicle services in V2X.

\section{VEHICLE ONTOLOGY}

In this section, we describe the proposed ontology which could be called vehicle ontology. To this end, we use the concept of the well-known OWL-S. The proposed vehicle ontology is summarized in Figure 2. As shown in Figure 2, the proposed ontology is composed of five models including VehicleService, Resource, ServiceProcess, ServiceProfile, and ServiceGrounding. The VehicleService model represents all of the vehicle services in V2X. The Resource model represents all of the resources which are used for enabling services. The ServiceModel represents a method to execute and operate regarding a specific service. The ServiceProfile model represents arguments for executing services. Finally, the ServiceGrounding model represents binding methods. More details are described in the next sections.

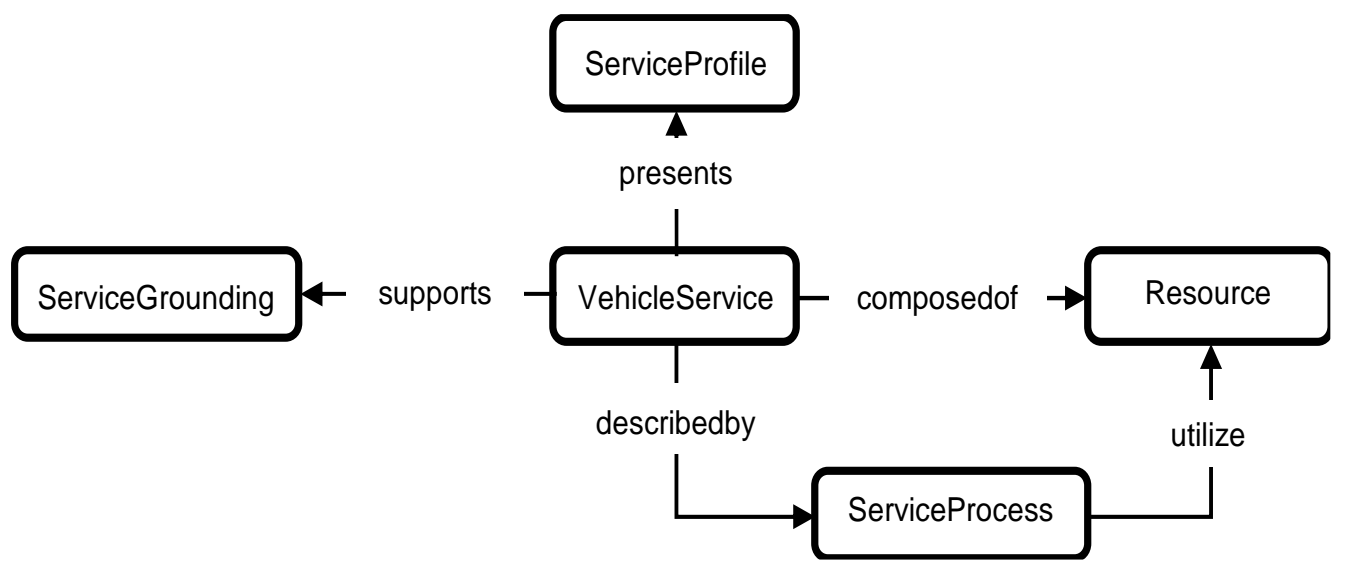

Fig. 2 The Vehicle Ontology which represents top-level ontology

\subsection{VEHICLESERVICE MODEL}

The VehicleService model describes general information for vehicle services in V2X. Accordingly, the VehicleService model includes classes of ServiceDescription, Type, and Owner as shown in Figure 3.

The ServiceDescription class represents a short description of a specific vehicle service where the description is expressed by hasDescription data property. The Type class represents communication categories in V2X such as V2V, V2I, V2P, and V2N. Hence, the type is expressed by the hasType data property. The Owner class represents ownership of a service. Each class has a relationship with the VehicleService class which is a superclass using hasSErviceDescription, hasServiceType, and hasOwnerID, respectively.

\subsection{RESOURCE MODEL}

The Resource model describes all of the resources which can be used to operate a service. In other words, all of the devices used in V2X are defined in this model. The Resource model includes three classes of ResourceDescription, ResourceType, and AccessInterface. The ResourceDescription class represents general information of the resource such as short description, access level, and whether it is a public resource or not. Each information is expressed by data properties of hasDescription, hasAccessLevel, and isPublic, respectively. The ResourceType represents the type of resource such as mobile phone, RSU, and OBU. Accordingly, each type is expressed by the hasType data property. Furthermore, to check the display device, hasDisplay data property is used in the class. Finally, the AccessInterface class represents information for the access point via the hasAccessPoint 
data property. Each class has a relationship with Resource class which is a superclass in the Resource model using object properties of hasResourceDescription, hasResourceType, and hasAccessInterface, respectively. Figure 4 shows the Resource model.

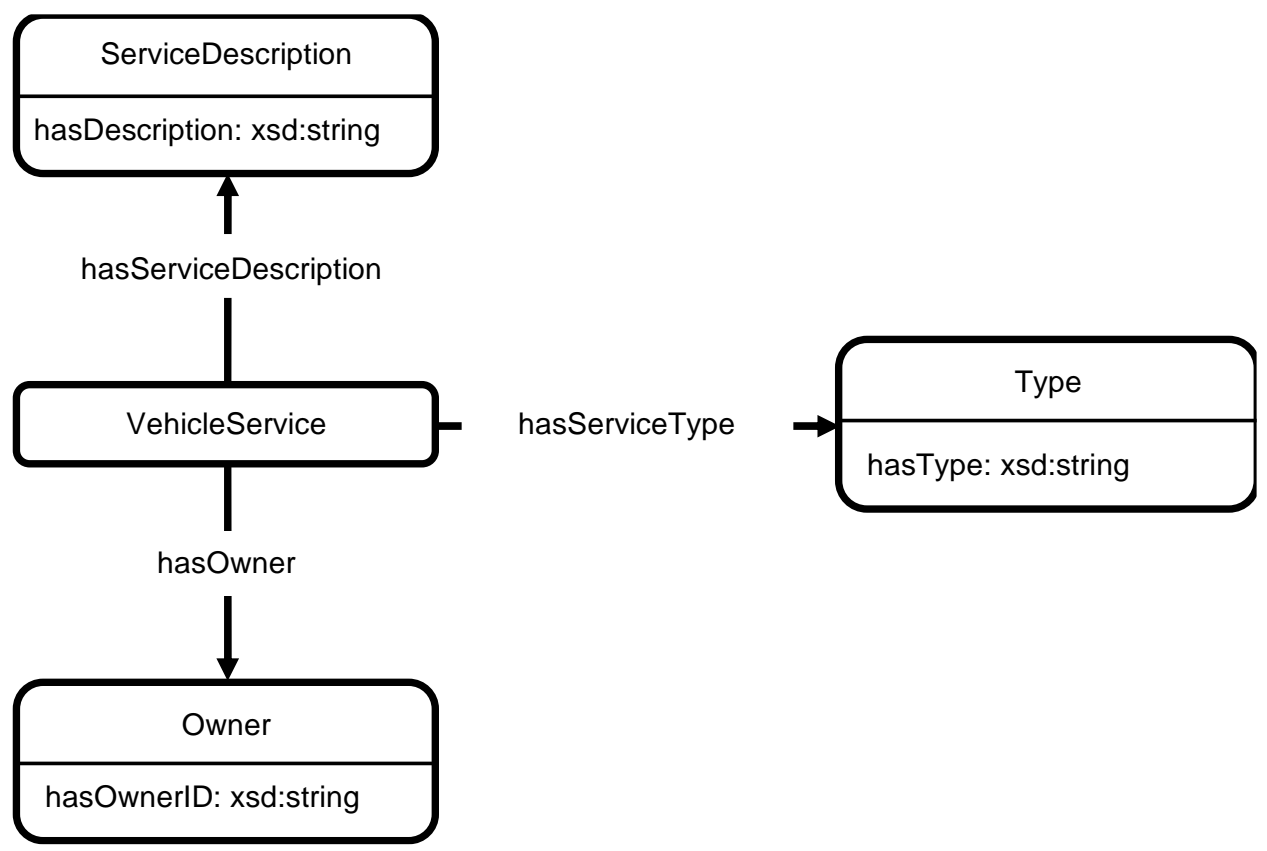

Fig. 3 The VehicleService Model

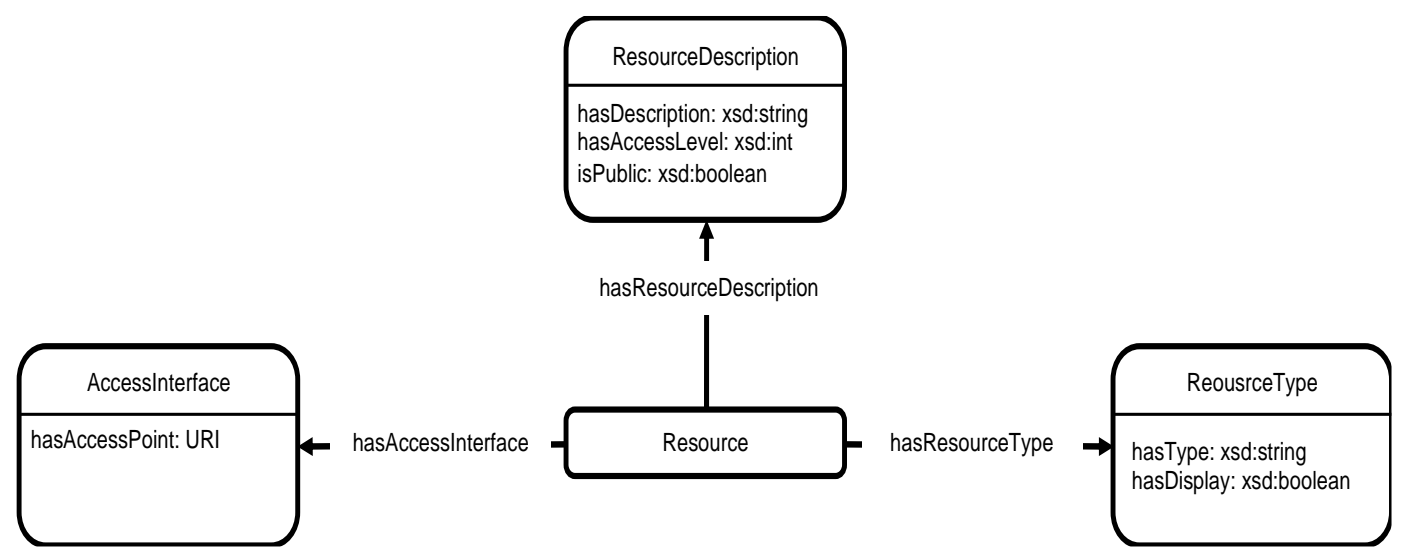

Fig. 4 The Resource Model

\subsection{SERVICEPROCESS MODEL}

The ServiceProcess model describes the method needed to execute a service defined in the VehicleService model. To this end, the model includes several classes to express the operating process of service as shown in Figure 5.

The ServiceProcess class is a superclass in the model and it has AtomicProcess class and SimpleProcess class as subclasses. In other words, one service process can be represented as an atomic process or simple process. Furthermore, each process can be represented as a composite process again. However, to represent the composite process, the service process should combine two more service processes. Accordingly, the SimpleProcess class has a relationship with the CompositeProcess class using object properties of expandsTo and collpaseTo, respectively. Also, the CompositeProcess class has a relationship with the ControlPlan class via the composedOf object property. The ControlPlan class represents controlling methods such as sequence, join, select, and split. Thus, each method is a 
subclass of the ControlPlan class. In addition, input, output, precondition, and effect which can be used to define processes are expressed as a class in the model. In the model, we comply with the original OWL-S ontological model instead of some classes.

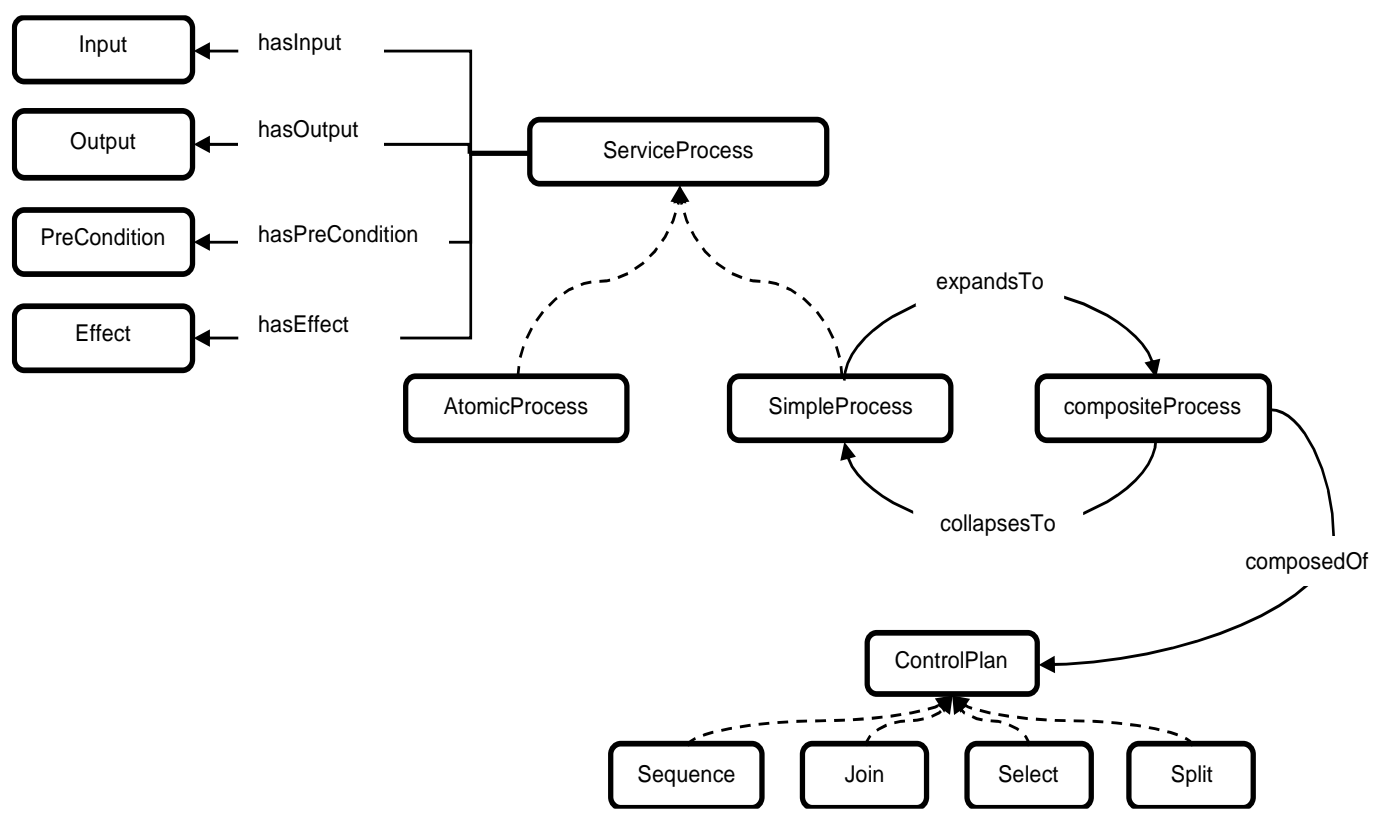

Fig. 5 The ServiceProcess Model

\subsection{SERVICEGROUNDING MODEL}

The ServiceGrounding model represents protocol binding to access services defined in the VehicleService model. Accordingly, the ServiceGrounding model includes two main classes such as Destination and StartingPoint. It also includes several protocol classes as shown in Figure 6.

The ServiceGrounding class is the superclass in the model and it has a relationship with other classes using object properties hasProtocol, hasDestination, and hasStartingPoint, respectively. In this model, we define the most popular protocol in V2X such as DSRC, Cellular, and Wi-Fi hotspot. In particular, we define Other class which can use to represent various protocols. Finally, through classes of Destination and StartingPoint, we can define the accessing target.

\subsection{SERVICEPROFILE MODEL}

The ServiceProfile model represents a profile of service defined in the VehicleService model where each service category is defined as shown in Figure 7. 


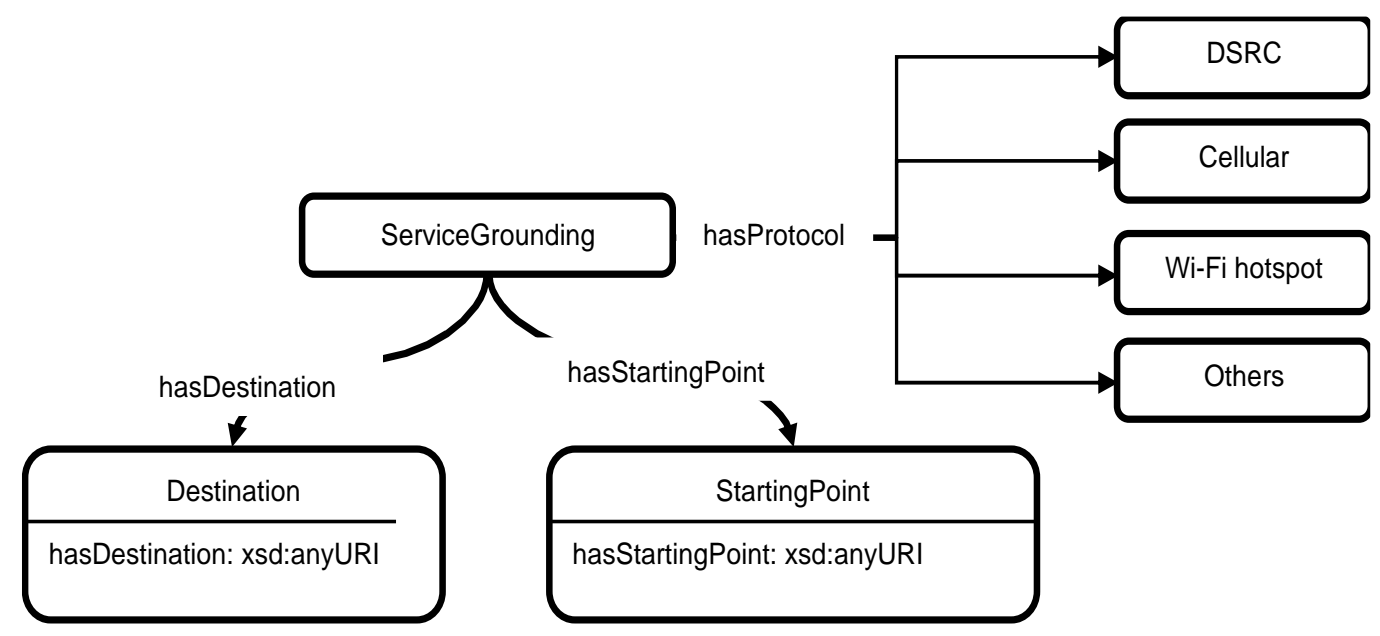

Fig. 6. The ServiceGrounding Model

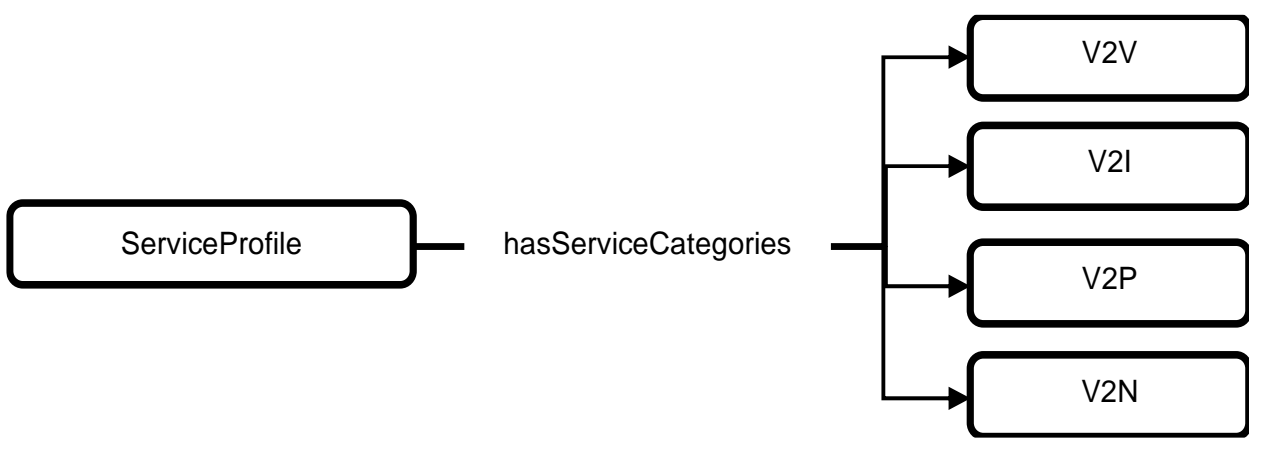

Fig. 7. The ServiceProfile Model

\section{IMPLEMENTATION}

In this section, we describe the implementation of the vehicle ontology using protégé and WebVOWL. The protégé tool is used to define classes, object properties, and data properties. In particular, we define domain and range regarding object properties in order to represent the relationship with each class. The WebVOWL tool is a visualization tool based on web browsers. It allows the display of an ontology as shown in Figure 8.

In Figure 8, the blue circles are represented as classes and blue rectangles are object properties. The yellow rectangles are represented as a type of data. The green rectangles are denoted by data properties. Finally, the dot arrow lines are represented as subclasses. As shown in Figure 8, each model is implemented as one ontological model. To this end, we define classes, object properties, and data properties as shown in Figure 9. 


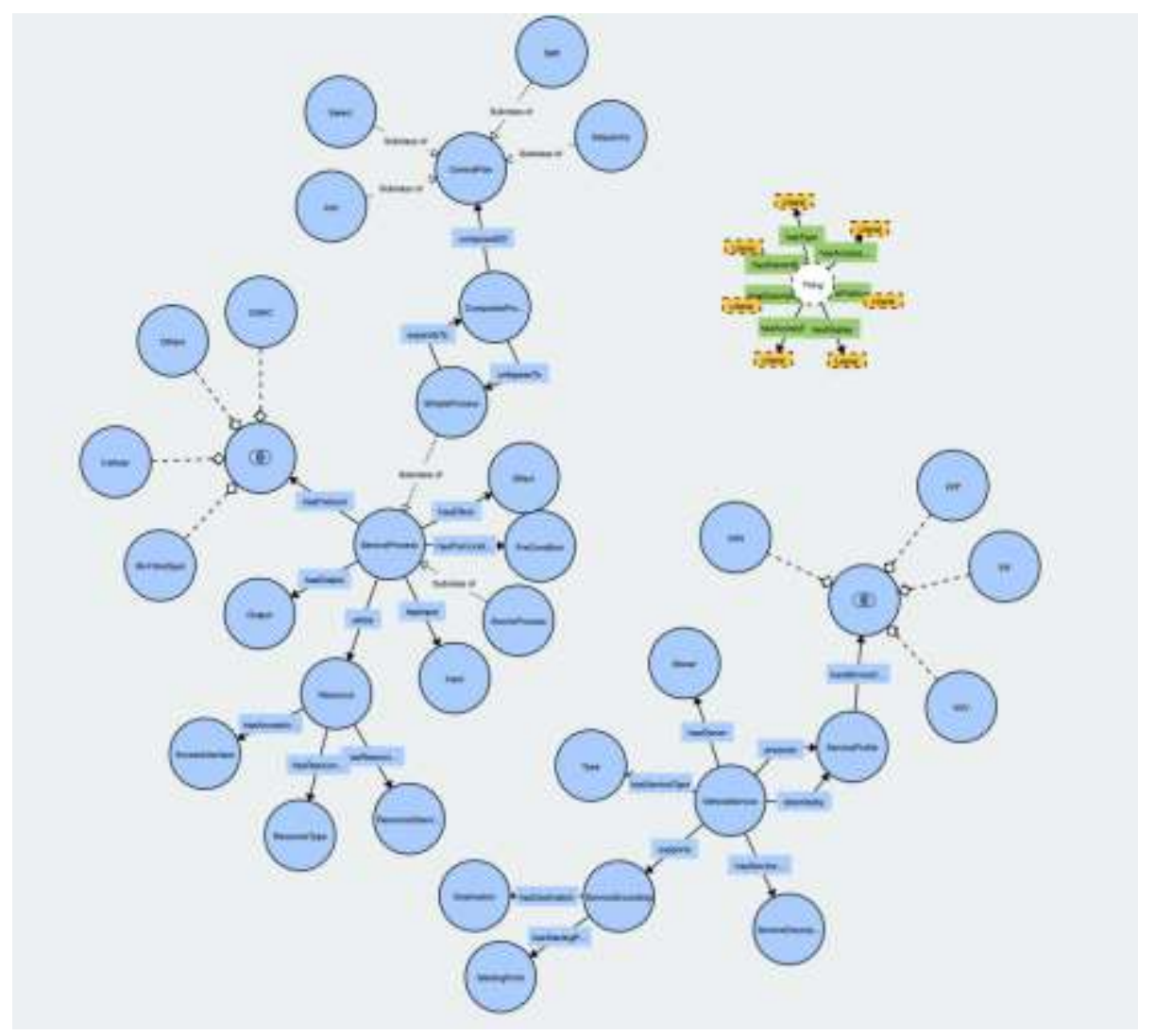

Fig. 8 Visualization of the vehicle ontology

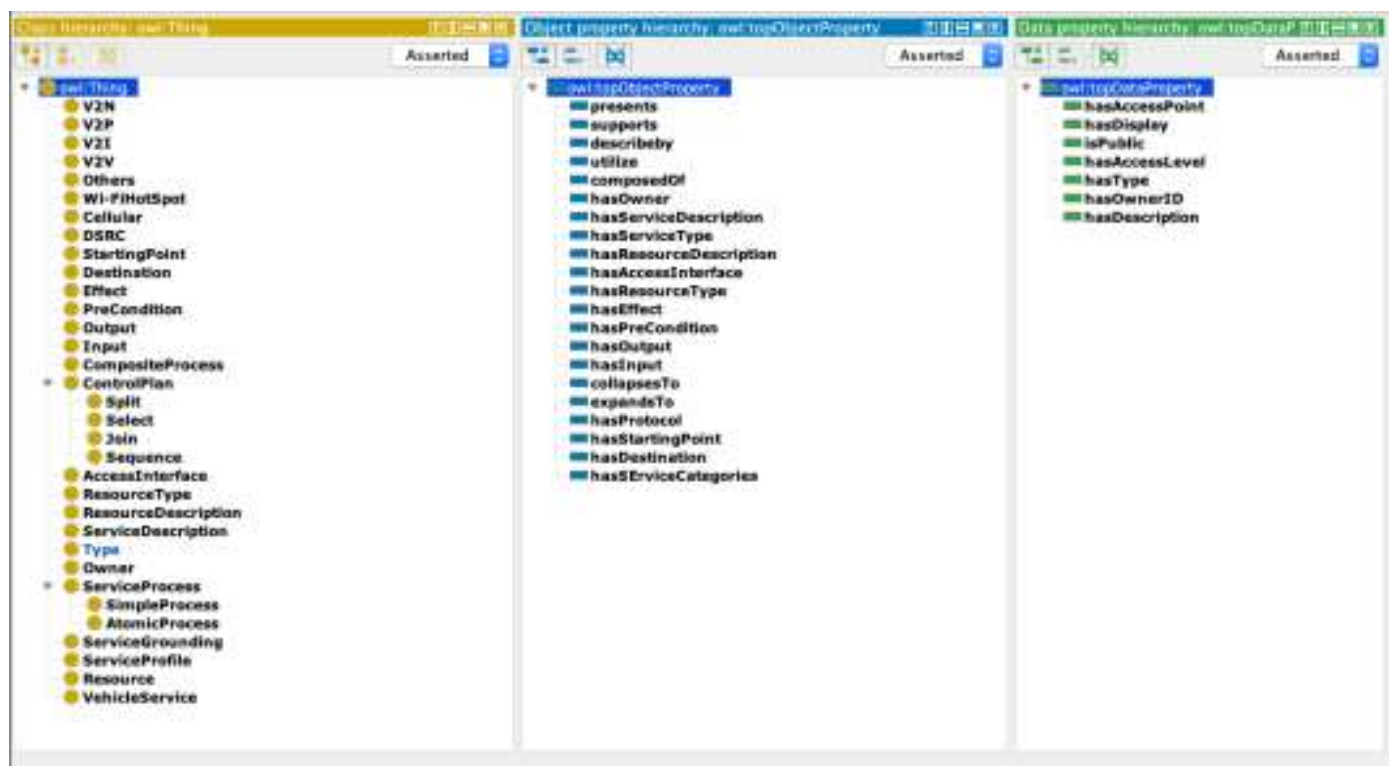

Fig. 9 The implementation of vehicle ontology using the protégé (from left) class hierarchy, object property hierarchy, and data property hierarchy

\section{CONCLUSION}

In this paper, we have presented a vehicle ontology based on well-known OWL-S in order to integrate various services in V2X. We designed and developed an ontology using the protégé tool. To this end, we designed and developed a five-sub ontological model including the VehicleService model, the Resource model, the ServiceProcess model, the 
ServiceProfile model, and the ServiceGrounding model. In particular, to guarantee ontological flexibility, we comply with OWL-S standard reparations. With the proposed ontological model, we have touched three main challenging issues as mentioned in Section 1. Furthermore, we show a visualization of the implemented ontology using the WebVOWL tool. Through threes results, we can conclude with confidence that the vehicle ontology can eventually contribute to the integration of various vehicle services in V2X communication networks.

Although we have provided vehicle ontology models for integrating various services in V2X communication networks, a system infrastructure is needed to manage the vehicle ontology. The vehicle ontology proposed in this paper can be used to understand and interpret data requested from vehicles. Accordingly, to handle and manage data, we need to consider the system infrastructure. Furthermore, the system infrastructure could resolve several issues by integrating some services in practice. Future research should be conducted to include a system infrastructure in order to manage the vehicle ontology according to data requested from vehicles.

\section{ACKNOWLEDGMENTS}

This research was supported by Basic Science Research Program through the National Research Foundation of Korea (NRF) funded by the Ministry of Education (NRF2016R1D1A1A09917662).

\section{REFERENCES}

[1] M. Ryu, S.-H Cha, J.-G. Koh, S. Kang, K.-H. Cho, "Position-based routing algorithm for improving reliability of inter-vehicle communication", Transitions on Internet and Information Systems (TIIS), 5.8 (2011).

[2] Eschenauer L., Gligor V.D, “A key management scheme for distributed sensor networks,” In proceedings of the $9^{\text {th }}$ ACM Conference on Computer and Communications Security, Washington, DC, (2002): 41-47.

[3] John B., and Kenney, "Dedicated short-range communications (DSRC) standards in the United States," In Proceeding of the IEEE 99.7 (2011): 1162-1182.

[4] A., Ghosh, R. Ratasuk, B. Mondal, N. Mangalvedhe, and T. Thomas, "LTE-advanced: next-generation wireless broadband technology," IEEE wireless communications 17.3 (2010): 10-22

[5] J. G. Andrews, S. Buzzi, W. Choi, S. V. Hanly, A. Lozano, A. C. Soong, and J. C. Zhang, "What will 5G be?," IEEE Journal on selected areas in communications 32.6 (2014): 1065-1082

[6] Di Zhang, "Distributed Cache Enabled V2X Networks, Proposals, Research Trends and Challenging Issues, arXiv preprint arXiv:1803.06059 (2018).

[7] Yi Wang, Akram Ahmed, Bhaskar Krishnamachari, and Konstantinos Psounis, "IEEE 802.11p performance evaluation and protocol enhancement," In 2008 IEEE International Conference on Vehicular Electronics and Safety, (2008).

[8] G. Anastasi, M. Conti, E. Gregori, and A. Passarella, "802.11 power-saving mode for mobile computing in Wi-Fi hotspots: limitations, enhancements and open issues," Wireless Networks 14.6 (2008): 745768.1

[9] D. Martin, M. Burstein, J. Hobbs, O. Lassila, D. McDermott, S. Mcllraith, and E. Sirin, "OWL-S: Semantic markup for web services,"' W3C member submission, 22.4 (2004).

[10] Toh, and K. Chai, "Ad hoc mobile wireless networks: protocols and systems," Pearson Education (2001).

[11] I.Q. Tse, "Improving Message Reception in VANETs," in Proceeding of International Conference on Mobile Systems, Applications and Services (MobiSys) (2009).

[12] S. H. Cha, J.-E. Lee, and M. Ryu, "Directed broadcasting with mobility prediction for vehicular sensor networks," International Journal of Distributed Sensor Networks 12.7 (2016): 1550147716657930.

[13] R. Amici, M. Bonola, L. Bracciale, A. Rabuffi, P. Loreti, and G. Bianchi, "Performance assessment of an epidemic protocol in VANET using real traces," Procedia Computer Science 40 (2014): 92-99.

[14] S. Zeadally, R. Hunt, Y.S Chen, A. Irwin, and A. Hassan, "Vehicular ad hoc networks (VANET): status, results, and challenges," Telecommunication Systems 50.4 (2012): 217-241.

[15] FA Silva, A. A Boukerche, T. R. Silva, E. Cerqueira, L. B. Ruiz, and A. A Loureiro, "Information-Driven Software-Defined Vehicular Networks: Adapting Flexible Architecture to Various Scenarios," IEEE vehicular Technology magazine 14.1 (2019): 98-107.

[16] D. Grewe, M. Wagner, S. Schildt, A. Nordmann, and J. Laverman, "Towards Semantic Object Discovery for Vehicular Named Data Networks," In 2018 IEEE 87 th Vehicular Technology Conference (VTC Spring), IEEE (2018). 
[17] M. Ruta, F. Scioscia, F. Gramegna, S. leva, E. Di Sciascio, and P. R.P De Vera, "A knowledge fusion approach for context awareness in vehicular networks," IEEE Internet of Things Journal 5.4 (2018): 24072419.

[18] J. Park, D. G. Yun. "The recognition prioritization of road environment for supporting autonomous vehicle," Journal of the Korea Academia-Industrial cooperation Society, 19. 2 (2018): 595-601.

[19] A. Bibi, O. Rehman, and S. Ahmed, "An Ontology based Approach for Messages Dissemination in Vehicular Ad Hoc Networks,” EAI Endorsed Trans. Scalable Information Systems 5.16 (2018): e2.

[20] Ginne Rani, Aman Dhingya, Ankur Gupta, Sagar Kumar, Swati Singh, "Implementation of Automatic vehicle accident detection system using Zigbee," International Journal of Science and Engineering for Smart Vehicles 1.1 (2017): 1-6.

[21] Jitesh Sah, Akshay Rawat, Manish Dhiman, Ankur Bhatt, Anoopshi Johari, "Smart Vehicle Monitoring System,” International Journal of Science and Engineering for Smart Vehicles 1.1 (2017): 7-14.

[22] Ch.Sekhar, S Satish.Effectively, "Utilization of Road Divider for Organized Vehicular Traffic using IoT," International Journal of Science and Engineering for Smart Vehicles 2.1 (2018): 1-10. 